\section{S13.3 PENILE MICROBIOTA, INFLAMMATION, AND HIV RISK}

Cindy Liu*. John Hopkins University, Baltimore, USA

10.1136/sextrans-2015-052270.63

Introduction The interplay between the genital microbiome and the host immune system may modify one's susceptibility to sexually transmitted infections. We examined the impact of circumcision on the penile microbiome and how it affects local immune response and potentially modifies the host's risk for HIV.

Methods Using real-time PCR, DNA sequencing, and Luminex multiplex assays, we studied the penile microbiome and levels of pro-inflammatory cytokines in the coronal sulcus of adult men who were randomized to immediate or delayed male circumcision in Rakai, Uganda.

Results Uncircumcised men frequently had high abundances of anaerobic bacteria, including Prevotella, Porphyromonas, and species of Clostridiales Family XI. Penile anaerobes decreased after male circumcision, replaced by low abundances of skinassociated bacteria such as Staphylococcus and Corynebacterium. Among the pro-inflammatory cytokines measured, the level of IL-8 correlated significantly with penile anaerobe abundance, which remained persistently elevated in uncircumcised men but decreased after circumcised men.

Discussion Our findings suggest that the penile microbiome may play a role in host susceptibility to HIV. Circumcision significantly decreased penile anaerobes and local production of proinflammatory cytokines, which may reduce HIV target cells recruitment and activation in the penile epithelium.

\section{S13.4 THE VAGINAL MICROBIOME AND STI}

Janneke van de Wijgert*. Liverpool University, Liverpool, UK

\subsection{6/sextrans-2015-052270.64}

Our systematic review of 63 molecular vaginal microbiome (VMB) studies conducted between 2008 and 2013 confirmed that lactobacilli-dominated $\mathrm{VMB}$ are associated with a healthy vaginal micro-environment and that bacterial vaginosis (BV) is best described as a polybacterial dysbiosis. However, not all lactobacilli seem to be equally healthy (e.g. a Lactobacillus inersdominated VMB is more likely to shift to dysbiosis than an $L$. crispatus-dominated VMB and L. iners often remains present during dysbiosis) and multiple dysbiosis entities with clinical relevance seem to exist. Some women with BV have a highly diverse $\mathrm{VMB}$ consisting of high loads of planktonic anaerobic bacteria, while others have a vaginal biofilm (typically including Gardnerella vaginalis). Furthermore, a small but clinically relevant proportion of women have a VMB dominated by Streptococci and/or Escherichia coli. Vaginal colonisation with Candida spp. is more common in women with a lactobacilli-dominated $\mathrm{VMB}$ than in women with dysbiosis, and women who receive antibiotic treatment for BV often subsequently develop vaginal candidiasis. Research has shown that all of these conditions cause disruption of the cervicovaginal mucosal barrier as well as cervicovaginal inflammation, which in turn might cause serious complications such as increased HIV acquisition, pre-term birth, and maternal/neonatal sepsis. At the moment, asymptomatic BV (by microscopic and clinical criteria) is usually not treated and symptomatic BV is treated with oral or vaginal metronidazole or clindamycine. However, recurrence rates are very high (up to $50 \%$ within six months) and novel treatments - such as vaginal biofilm disruption and vaginal probiotics - are therefore needed. Furthermore, the different dysbiosis entities, or combinations of entities, likely require different clinical management approaches. Systems biology has now become more affordable and should be incorporated into epidemiological studies to address associations of different dysbiotic entities with clinical outcomes, and to evaluate interventions aimed at restoring and maintaining a lactobacilli-dominated VMB.

\section{S14 - Causal interactions among structural, behavioural and biological drivers of STD/ HIV epidemics}

\section{S14.1 TRANSITIONS AROUND SEX WORK AND THEIR SIGNIFICANCE IN STI/HIV EPIDEMICS}

Marissa Becker*. University of Manitoba, Winnipeg, Canada

\subsection{6/sextrans-2015-052270.65}

In many regions of the world, the importance of formal sex work in driving HIV epidemics is well-established and it is recognised that female sex workers (FSWs) experience a disproportionately high risk of HIV globally. Sex work and sex work networks are complex and continuously evolving and therefore FSW research needs to address this changing nature of HIV risk as well as the contribution of other important factors to the overall HIV epidemic, such as macro-structural determinants, sub-population-level social and sexual networks, individual behaviours and host and viral biological factors. Further, early HIV and STI risk has been particularly identified as being an important area of programmatic and research focus. In an effort to understand the complexity and risk trajectory associated with sex work, the "Transitions" study examines the HIV risk and vulnerability among young women and FSWs over the life course from the structural, network, behavioural and biological perspectives. Transitions also aims to dissect the contribution and interaction of factors within these different areas (structural, network, behavioural and biological) that drive HIV risk and transmission at an individual- and population-level. Disentangling the role of these contributing factors in HIV risk could shed light on the optimal mix of HIV interventions that is proportionate to the relative influence of these drivers.

This presentation will introduce a framework that demonstrates how Transitions brings together the various perspectives in its work in the different epidemiological contexts of Ukraine and Kenya. For the latter, mapping results from Ukraine and Kenya will be reviewed. This presentation will also show how such a framework is used to guide research and inquiry in order to generate meaningful and concrete findings that could have implications on HIV prevention and control programming.

\section{S14.2 AGE, SEXUAL EXPERIENCE AND MUCOSAL INFLAMMATION: WHAT DO WE KNOW?}

Lyle McKinnon*. University of Manitoba, Winnipeg, Canada

\subsection{6/sextrans-2015-052270.66}

HIV transmission rates are low on a per-coital level, but increased by various transmission co-factors. One implication of 